\title{
Possible phase separation in square and honeycomb Hubbard model: a variational cluster study
}

\author{
Kun Fang \\ Department of Physics, University of Connecticut, Storrs, CT 06269, USA \\ G. W. Fernando \\ Department of Physics, University of Connecticut, Storrs, CT 06269, USA
}

A. V. Balatsky

Los Alamos National Laboratory, Los Alamos, NM 87545, USA

A. N. Kocharian

Department of Physics, California State University, Los Angeles, CA 90032, USA

\begin{abstract}
The VCA ground state of the 2D Hubbard model is examined for possible phase separation under hole doping manifested by spatial inhomogeneities of coexisting different electron densities at equilibrium. Phase separation is accompanied by spectral weight loss and first Brillouin zone boundary deformation. Such instability is observed in square structures and it is absent in honeycomb lattices. To our knowledge, no previous publications have revealed relationship between Fermi surface instability and phase separation. Our VCA calculations provide strong support for spontaneous instability, driven by electron correlations in specific lattice geometries, proposed in our earlier publications using exact quantum cluster calculations.
\end{abstract}

Key words:

VCA; quantum cluster calculation; phase separation; nanoscale inhomogeneities; square and honeycomb lattices; Brillouin zone deformation

PACS: 71.10.-w, 73.22.Gk, 74.20.-z, 74.72.-h

Email address: armen.kocharian@calstatela.edu (A. N. Kocharian). 


\section{Introduction}

One of the most challenging problems in condensed matter physics has to do with understanding the physics of strongly correlated materials. Such electronic materials, found (for example) in certain oxides and selenides, give rise to a broad range of different phenomena and display complex phase diagrams. Electronic inhomogeneities originated from strong correlations have been discovered in the cuprates and iron-based superconducting materials. These spatial inhomogeneities, also often called phase separation (PS), are present as nanoscale spatial variations of electron densities in the form of stripes[1], "checkerboard" [2] and granular structures[3,4]. Theoretical investigations of strongly correlated materials are based on numerical calculations of interacting electron lattice models and most specifically are focused on the Hubbard model [5]. Recently, various numerical methods have been employed to provide insights into the many-body physics of the model, such as exact diagonalization (ED), quantum Monte Carlo (QMC)[6], dynamical mean field theory (DMFT)[7], dynamical cluster approach (DCA)[8] and variational cluster approximation (VCA) [9].

The importance of electronic phase separations driven by electron correlations was realized in the Hubbard model by Visscher[10] in the 1970s and was systematically studied at the strong interaction limit later using the $t-J$ model $[11,12]$. Our recent many-body calculations in different bipartite and non-bipartite clusters on phase separation in the Hubbard model are based on exact diagonalization[13-17]. These results at some critical Coulomb interactions display a level crossing instabilities similar to phase separation transitions seen at various doping levels even close to the optimal doping[1-3]. However, in spite of high accuracy cluster calculations always are inevitably tied to some uncertainties due to size and edge effects. Macridin et al.[18,19] proposed a thermodynamical description of electronic phase transition, and the theory further points to the existence of a quantum critical point which closely resembles the level crossing point observed near phase separation instability at the cluster level in Refs. [13-15]. Aichhorn et al.[20,21] discussed the coexistence of the global antiferromagnetic and superconducting long-range orders in variational cluster calculations. However, STM experiments show strong evidence that at very low temperature, electronic states on BSCCO is inhomogeneously distributed and there is no sign of any long-range ordering[3]. Therefore, here we exclusively focus on direct effects of inhomogeneities in the 2D Hubbard model at zero temperature using VCA method as an accurate variational tool to incorporate a cluster-cluster coupling in two-dimensional (2D) Hubbard lattice without any assumption on possible long-range ordering. Recent discoveries suggest that phase separation is likely to occur in the vicinity of a metal-insulator transition (MIT)[22-24]. In contrast to onedimensional systems, the 2D Hubbard model in both square and honeycomb 
lattices at half-filling is expected to exhibit a Mott-Hubbard MIT at finite on-site Coulomb interactions $U$ in the paramagnetic phase at both zero and finite temperature[25-30]. In general, this transition at a finite $U$ value might be an intrinsic property of the 2D Hubbard lattice[30]. Historically, there have been contradictory claims with regard to phase separation in the 2D Hubbard model. In early QMC work [31,32], it was concluded that there was no sign of phase separation in the range of the parameters studied. Certain analytical estimates on the 2D Hubbard model by Su [33] appear to suggest that no phase separation exists at finite temperature. Some of the limitations in these approaches may have led to such conclusions. However, more recent work, using the DCA[18], has captured a phase separated region consisting of coexisting strongly correlated metallic and Mott insulating phases.

In this work, we investigate possible phase separation in both square and honeycomb lattices from half-filling to optimal doping at zero temperature following the VCA procedure. This method is known to be accurate when electron correlations are local, which is quite likely in the case of high temperature superconductors in the absence of long-range order. We find strong evidence of phase separation (co-existence of different phases) for the square lattice in the underdoped region. However, no phase separation is found in the honeycomb lattice within a similar region of doping and Coulomb interactions. Some of the important outputs of the method, such as the one-particle excitation spectral functions and spin susceptibilities in the relevant doping region, are used to identify a possible geometry-related mechanism for phase separation. The rest of the paper is organized as follows: In the following section, we formulate (in a nutshell) the principles of exact quantum cluster calculations and present the basic methodology of the variational cluster approximation. In Sec. 3, we study the MIT at half-filling and phase separation effects away from half-filling in square and honeycomb structures to underline their differences observed already at the cluster level. The concluding remarks are given in Sec.4.

\section{Method}

\subsection{Hamiltonian}

We use one-band Hubbard Hamiltonian $\hat{H}$ :

$$
\hat{H}=\sum_{\left\langle\boldsymbol{r}, \boldsymbol{r}^{\prime}\right\rangle \sigma}\left(-t \hat{c}_{\boldsymbol{r} \sigma}^{+} \hat{c}_{\boldsymbol{r}^{\prime} \sigma}+H . c .\right)+U \sum_{\boldsymbol{r}} \hat{n}_{\boldsymbol{r} \uparrow} \hat{n}_{\boldsymbol{r} \downarrow}
$$


where $\hat{c}_{\boldsymbol{r} \sigma}$ is the annihilation operator for electrons at site $\boldsymbol{r}$ with spin projection $\sigma$ and $U$ is the on-site screened Coulomb repulsion. $\left\langle\boldsymbol{r}, \boldsymbol{r}^{\prime}\right\rangle$ denotes summation over the nearest neighbors. The coupling parameter $t$ is the transfer integral between the nearest neighbors. All the energies reported here are measured in units of $t>0$. We focus here on the case with hole doping, when the electron number per atomic site $\left(n=N / N_{a}\right)$, i.e., electron density $n<1$.

\subsection{Quantum cluster calculations}

The thermodynamic and ground state properties of the Hubbard model with a large number of electrons and lattice sites cannot be calculated by exact diagonalization technique. However, full diagonalization is still possible within relatively small Hubbard clusters such as squares, 8 -site $2 \times 4$ ladders, twodimensional Betts cells and pyramids[34,35].

The problem of a quantum gas of interacting many electrons in independent clusters is exactly solvable in the ground state and at finite temperatures [1317]. The canonical and grand canonical ensembles in equilibrium display thermodynamic phase diagrams and the tendency to phase separation through responses of the system to changes of electron concentration and magnetic field. For instance, the charge excitation gap, $\Delta^{c}(U)=E(N+1)+E(N-1)-2 E(N)$, is calculated using the lowest canonical energies $E(N)$ with the number of electrons $N$ in a particular doping region close to the half-filling. The positive gap region at relatively large $U$ and one hole off half-filling describes a Mott Hubbard insulator. The negative excitation gap $\Delta^{c}<0$ earlier found in various $2 \times 2$ and $2 \times 4$ square geometries displays an electron charge instability at weak and moderate $U$ values. The nodes of the charge gap, i.e., $U$ values at which $\Delta^{c}(U)=0$, determine the critical parameter $U_{c}$ for possible level crossing instabilities. This implies a redistribution of electron density and phase (charge) separation (i.e., segregation) of the clusters into hole-rich and hole-poor regions for different numbers of electrons per cluster. The inhomogeneities favored by the negative gaps are essential for generating the spontaneous electron charge separation. Here we show the contrasting behavior of a charge energy gap versus $U$ in bipartite honeycomb geometry at one hole off half-filling. In Fig. 1, the gap opens at infinitesimal $U$ and increases with $U$ monotonically without charge phase separation by avoiding level crossings. This positive charge gap observed at the cluster level is crucial for stabilization of homogeneous electron density in the large honeycomb lattice. Our VCA calculations in honeycomb lattice at infinitesimal $U$ in close vicinity of half-filling confirm such insulating behavior in Fig. 1 at one hole off half-filling as $U \rightarrow 0$. 


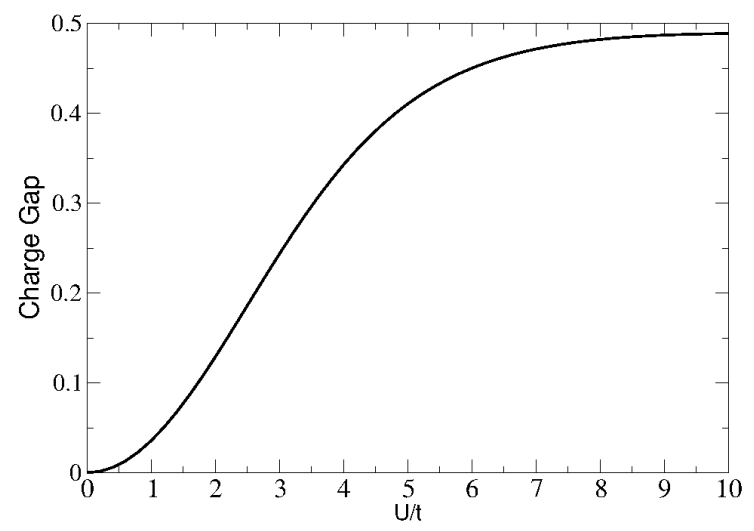

Fig. 1. The charge energy gap variation as a function of on-site interaction strength $U$ in the 6-site honeycomb cluster. There is no sign of phase separation instability driven by $U$, since the gap opens at infinitesimal $U$ and increases monotonically.

One can extrapolate the results of many-body cluster calculations obtained for small clusters to larger two-dimensional lattices and consider the "concentrated" two-dimensional Hubbard lattice modeled as a checkerboard lattice structure with embedded arrays of such disconnected unit cells, which do not interact directly, but form a grand canonical ensemble in thermodynamic equilibrium. In inhomogeneous concentrated systems, this description in thermodynamic equilibrium becomes quite accurate for suitable values of parameters since the lattice can be broken up into periodic arrays of weakly coupled clusters. In the VCA, the cluster-cluster interaction is usually added through the coupling between the unit cells comparable to the energy transfer scale $t$ within an individual cluster. The VCA provides accurate results for cluster sizes not accessible to full diagonalization. Thus, exact predictions given by a grand canonical ensemble of clusters in various geometries can be tested using the VCA results in the "concentrated" 2D Hubbard lattice. Below we compare the geometry dependence in square and honeycomb bipartite lattices using the disconnected square and honeycomb clusters of lattice sites (that we solved exactly) in the VCA as a reference system.

\subsection{Variational cluster approximation}

As mentioned earlier, the VCA [9] is used to solve the Hubbard model on a large 2D lattice. This is a quantum cluster[8] extension of Potthoff's selfenergy functional approach (SFA)[36]. Unlike some Monte Carlo methods, the VCA accompanied with exact diagonalization does not encounter any difficul- 
ties, such as the sign problem, at low temperature or doping near half-filling. The VCA method decomposes the lattice (original system) with one-particle parameters $\boldsymbol{t}$ and on-site interaction parameter $U$, into isolated, small clusters (reference system) with modified one-particle parameters $\boldsymbol{t}^{\prime}$ and the same on-site interaction parameter $U$. The Hubbard model on the cluster is exactly solvable by either exact diagonalization[13] or Lanczos method[37]. The exact eigenvalues and eigenvectors can lead to an accurate implementation of the correlation effects through the self-energy $(\boldsymbol{\Sigma})$. The method provides sufficient accuracy of short-range correlation effects in self-energy because it becomes exact at the cluster level when coupling parameter between clusters tends to zero. The exact self-energy in the reference system is used to approximate the self-energy of the original infinite system, while the hopping parameters between clusters are treated by cluster perturbation theory[38]. The grand potential $\Omega$ of the original system can be written as a functional of the selfenergy $\Sigma$ provided the system is not at a critical point associated with a phase transition[36]:

$$
\Omega_{\boldsymbol{t}}[\boldsymbol{\Sigma}]=\Omega_{\boldsymbol{t}^{\prime}}[\boldsymbol{\Sigma}]-\operatorname{Tr} \ln \left(\boldsymbol{G}_{0}^{-1}-\boldsymbol{\Sigma}\right)+\operatorname{Tr} \ln \left(\boldsymbol{G}_{0}^{\prime-1}-\boldsymbol{\Sigma}\right)
$$

where $\boldsymbol{G}_{0}$ and $\Omega_{t}$ are the non-interacting Green's function and the grand potential of the original system while the respective primed variables are for the reference system. The above functional is proved to be stationary at physical self energy $\partial \Omega / \partial \Sigma=0$, so the ground state of the system can be obtained by locating the stationary point in the self energy space. A straight forward way to find the stationary point is using the variational calculation (not a selfconsistency calculation) based on the above equation, by varying one-particle terms $\boldsymbol{t}^{\prime}$. Ref. [36] has provided rigorous proof that $\Omega[\boldsymbol{\Sigma}]$ is stationary at the ground state without introducing a long-range order through the Weiss fields. However, because the evaluation of the stationary points of the functional is performed in the restricted self-energy space of the reference system, instead of the full self-energy space, the ground state found in the VCA is still approximate.

In this study, we apply the VCA method with reference systems as shown in Figs. 2 and 7 to derive the ground state properties of square and honeycomb lattices at zero temperature. We do not introduce any long-range orders so the Hamiltonian at the cluster level has the same form as the original Hamiltonian of the lattice. The variational calculation is performed at the cluster level on one-particle parameters, $t^{\prime}$ and $\mu$. The size of the reference system (i.e., the size of the isolated clusters) determines the dimensionality of the self-energy space in the variational calculation; hence, in principle, it affects the accuracy of the results. Below, in all our calculations we use $15 \times 15$ lattice size for the original system. In practical calculations, the improvement on the accuracy gained by enlarging cluster size does not significantly affect results of both 
energy and one-particle excitation spectrum $[9,39]$ of the 2D systems discussed here.

\subsection{Spontaneous phase transition}

Recent STM studies[3] have confirmed that at low temperature, electronic states are inhomogeneously distributed throughout the cuprate materials. This suggests that, under such conditions, long-range orders do not play an important role. In order to study the effects caused purely by inhomogeneous states, we apply the VCA calculations to the 2D Hubbard model without introducing any long-range fictitious fields at the cluster level.

Spontaneous phase separation, seen in our zero-temperature VCA calculations at moderate $U$ values, describes an inhomogeneous distribution of electron densities in the lattice, i.e., states with different electron densities in equilibrium can simultaneously coexist in the lattice. According to phase transition dynamics, the chemical potentials $\mu$ of different states with electron densities $n$ have to be the same in the system[40] at equilibrium. Therefore, if $n$ can be obtained as a function of $\mu$, a multivalued $n$ at certain $\mu$ values can be considered as clear evidence of an electronic phase separation instability.

\section{Results}

\subsection{Square lattice}

\subsubsection{Half-filling}

As mentioned in Sec. 1, the MIT might be relevant to phase separations and hence we study the MIT at half-filling first. The one-dimensional Hubbard model leads to a smooth MIT at infinitesimal $U$ values and shows no phase separation at any $U$ value. In two dimensions, our VCA calculations for the square and honeycomb geometries provide strong support for a smooth second order Mott-Hubbard MIT at half-filling. We found that a finite Coulomb interaction $U=U_{c}>0$ is needed to open an energy gap around the Fermi level in the one-particle excitation spectra for both (square and honeycomb) lattices. The critical $U_{c}$ value at the transition is approachable from both sides of $U_{c}$. In the square lattice, the MIT transition takes place approximately at $U_{c}=1.5$ while in the honeycomb lattice it is about $U_{c}=2.5$. We would like to emphasize that since we do not include any long-range magnetic order (by adding Weiss field parameters to reference systems), and, therefore, our results 
reflect intrinsic properties of the 2D Hubbard model in the VCA calculation at the absence of any long-range order.

\subsubsection{Phase separation}

Phase separation, caused by electron instabilities under hole doping, leads to the coexistence of inhomogeneous, hole-rich and hole-poor regions. This phenomenon closely resembles phase separation instabilities observed in first order phase transitions. The coexisting states with different electron densities $n$ share the same chemical potential $\mu$. The $n$ vs $\mu$ plots, shown in Fig.2, are obtained for the square lattice at different Coulomb interactions $U$ from our VCA calculation. It is found that the plots for the square lattice show discontinuities where the dependence of $n$ on the chemical potential $\mu$ is multivalued. This implies that electronic phase separation occurs in the square lattice at the vicinity of such discontinuities which is similar to the classic spontaneous phase separation in a first order phase transition. For example, at $U=4$, the discontinuity of the plot appears at $\mu$ around 1.25 and the value of $n$ changes abruptly from 0.946 to 0.977 . These two states have almost the same chemical potential and, therefore, they can coexist in the system. When the lattice has an average electron concentration between 0.946 and 0.977 , the lattice system will become phase separated and behave as a mixture of hole-rich $(n=0.946)$ and hole-poor $(n=0.977)$ regions, which leads to spatial inhomogeneities.

As $U$ becomes larger, the magnitude of the discontinuity in $n$ also increases (see Fig. 2), which suggests that the electron-electron interaction plays an important role in the electronic phase separation. In addition, since no long-range order term (for spontaneous symmetry breaking) has been added in the variational calculation, the ground state is driven purely by local electron correlations. Electronic phase separation occurs at some finite Coulomb interaction strength $U_{c}$, which is close to the MIT transition value at half-filling. This result suggests that the electronic phase separation in the square lattice is closely related to the Mott-Hubbard transition and is likely to be driven by the onsite Coulomb interaction. Similar results were also reported in Ref.[18] using the DCA. However, in our previous study of isolated clusters[13-17], we found that the abrupt change of density in Fig. 2 decreases by increasing temperature. The discontinuity gradually disappears and becomes a smooth crossover at relatively low finite temperature (about $0.15 t$ at $U=4$ in Ref. [17]). DCA calculation uses Quantum Monte Carlo method which is limited to finite temperatures so it is impossible to compare their calculations performed at finite temperature with ours results at zero-temperature. Our previous studies also find that the introduction of the next-nearest-neighbor hopping reduces the discontinuity (in Fig. 2), i.e., abrupt change in electron density, and smoothen the transition only at negative $t_{n n n}<0$, while for positive $t_{n n n}>0$ value this discontinuity can become even larger (see Ref. [17]). 


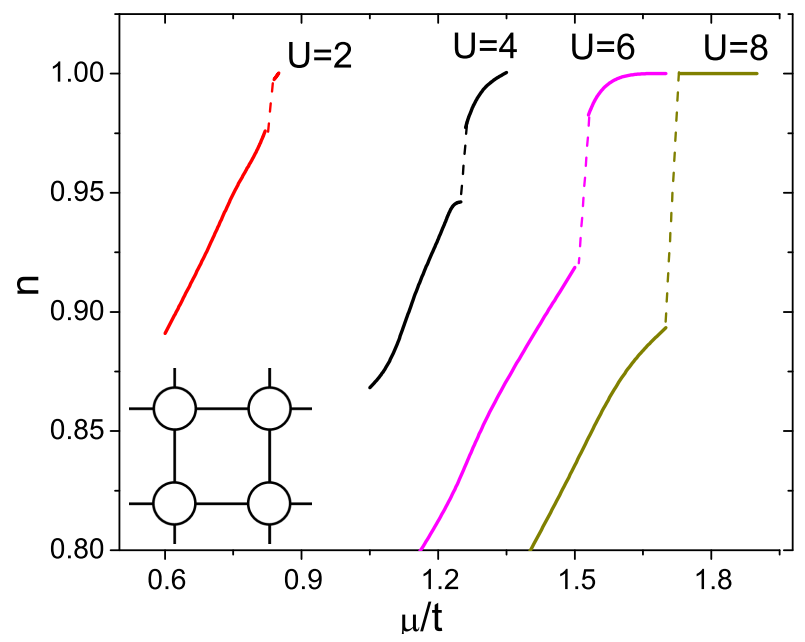

Fig. 2. The electron densities $n$ as a function of chemical potential $\mu$ for the Hubbard model on a square lattice at different values of on-site Coulomb interaction $U$. The plots clearly show discontinuities at distinct $n$ values under hole doping. The discontinuity means that states with different $n$ at two edges of the step-like discontinuous transition have the same $\mu$ value, so they can coexist in the same lattice at the equilibrium, which provides a spatial variation of charge density in the material. The magnitude of the discontinuity of $n$ becomes larger as $U$ increases, which indicates that the phase separation is preferred when interaction gets stronger. The reference system (2x2 cluster) of the VCA calculation is shown in the inset.

There are questions related to the sizes of the AF or metallic domains when the system is phase separated. As discussed in Sec 1, experiments show that the length scale of inhomogeneities has a very wide range. Theoretically, the length scale should be determined by the interaction strength and correlation length in the model[12]. However, the correlations are restricted to the cluster size in the VCA and hence it is not possible to address this question directly from our calculation.

\subsubsection{Antiferromagnetic transition}

The similarity between this electronic phase separation found in the square lattice and the classic phase separation found in the first order phase transitions suggests that it is very likely that there is a phase transition when electronic phase separation happens. It is known that the lattice is antiferromagntic (AF) at half-filling and it becomes a metal with sufficient hole doping. The electronic phase separation found in the previous section may reflect a phase transition between the AF state and the metallic state. Although our VCA calculation does not include any long-range order, for example longrange AF order, some AF correlations may still appear locally at the cluster length scale because many-body effects are treated exactly at the cluster level in the VCA calculation. In order to confirm whether their is an AF-normal 
metal transition when there is a phase separation, we analyze the transverse spin susceptibility $\chi$ defined as

$$
\chi_{i j}(\omega)=\int_{0}^{\beta} d \tau e^{i \omega \tau}\left\langle\hat{S}_{i}^{+}(\tau) \hat{S}_{j}^{-}(0)\right\rangle
$$

with

$$
\begin{aligned}
& \hat{S}_{i}^{+}(\tau)=\hat{c}_{i \uparrow}^{+}(\tau) c_{i \downarrow}(\tau) \\
& \hat{S}_{i}^{-}(\tau)=\hat{c}_{i \downarrow}^{+}(\tau) c_{i \uparrow}(\tau)
\end{aligned}
$$

When calculating $\chi(\boldsymbol{k}, \omega)$, we only count up to the zero-order diagram $\chi^{0}(\boldsymbol{k}, \omega)$ and ignore all the higher order terms which take into account the non-irreducible two-particle vertex $\Gamma(\boldsymbol{k}, \omega)$. Although $\Gamma(\boldsymbol{k}, \omega)$ contains important information about dynamical correlations, the $\chi^{0}(\boldsymbol{k}, \omega)$ is sufficient to be a "fingerprint" of a simple static AF local correlation with an AF vector $\boldsymbol{Q}=(\pi, \pi)$. Therefore, the transverse spin susceptibility in momentum space can be obtained from the one-particle Green's function $\boldsymbol{G}(\boldsymbol{X} ; \boldsymbol{k}, \omega)$ calculated by the VCA method as

$$
\chi(\boldsymbol{k}, \omega) \approx \chi^{0}(\boldsymbol{k}, \omega)=\frac{1}{N} \sum_{\boldsymbol{q}} \int \frac{d \nu}{2 \pi} \boldsymbol{G}_{\uparrow \uparrow}\left(\boldsymbol{X}^{\prime} ; \boldsymbol{k}+\boldsymbol{q}, \omega+\nu\right) \boldsymbol{G}_{\downarrow \downarrow}(\boldsymbol{X} ; \boldsymbol{q}, \nu)
$$

where $\boldsymbol{X}$ in the one-particle Green's function denotes a real space vector between two cluster sites and $N$ is the number of $\boldsymbol{k}$-points used in the calculation. For the present work, $N=15 \times 15$ has been used which is directly related to the size of the original system.

We compare $\chi$ around the $(\pi, \pi)$ point in the $k$-space for different electron concentrations. The results for $U=4$ are plotted in Fig. 3 at half-filling $(n=1)$ for the two states right before and after phase separation $(n=0.977$ and $n=0.946)$. At half-filling, there is a high susceptibility at the $(\pi, \pi)$ point, which indicates a strong (saturated) AF correlation. This characteristic peak in the momentum space at vector $\boldsymbol{Q}=(\pi, \pi)$, corresponds to doubling of lattice period at short-range (local) AF correlations in a real space. At $n=0.977$, there is still a small peak, so a relatively weaker AF correlation still exists at this concentration. While the system goes through the phase separation region, the AF correlations are destroyed since no peak is seen at the $(\pi, \pi)$ point for $n=0.946$. This plot clearly shows that when the electron density drops from 0.977 to 0.946 , the AF correlations become weak before vanishing eventually. 


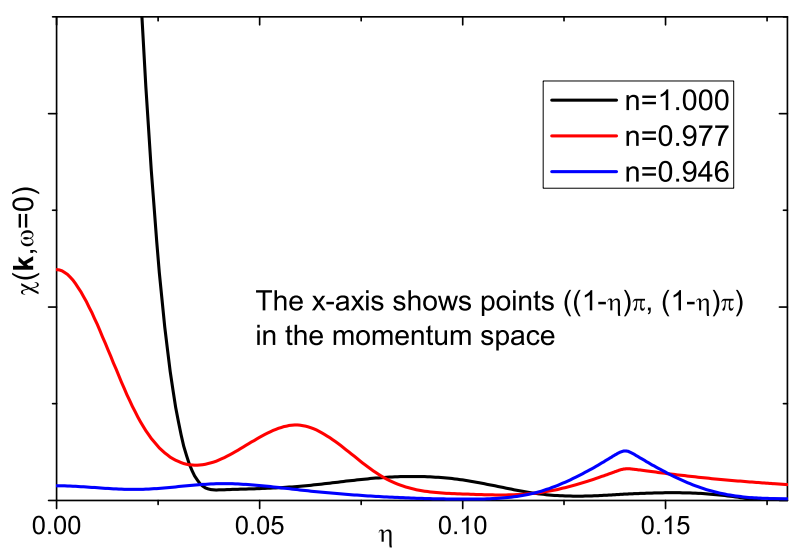

Fig. 3. The zero-frequency transverse spin susceptibilities $\chi(\boldsymbol{k}, \omega=0)$ at $U=4$ with different electron densities are obtained along $(\pi, \pi)-(0,0)$ direction. The $\mathrm{x}$-axis denotes the $\boldsymbol{k}$ point at $((1-\eta) \pi,(1-\eta) \pi): \chi(\boldsymbol{k}, \omega=0)$ has a huge peak at $(\pi, \pi)$ which is the "fingerprint" of a static local AF correlation. This peak remains but gets smaller when $n=0.977$, which indicates that the local AF correlation can still be observed but it is not very strong. As the system goes through the phase separation region to a lower density $(n=0.946)$, the susceptibility peak disappears indicating that the AF correlation is totally destroyed. The results are derived from VCA calculation using $2 \times 2$ cluster as the reference system. A $15 \times 15 \mathrm{k}$-space grid is used for k-space integration.

\subsubsection{Phase diagram}

A hole concentration $x$ versus $U$ phase diagram for the square lattice can be extracted from the above calculations as shown in Fig. 4. The metallic (M), antiferromagnetic insulator (AFI) and phase separation regions are identified in the above figure. This figure indicates that the square lattice has a phase separation region, in which the system is an inhomogeneous combination of a metal and an AF insulator. The range of change in the electron density (discontinuity) at phase separation increases when $U$ becomes larger, which indicates that the AF correlation plays an important role in generating the electronic phase separation. At half-filling, as long as $U$ is larger than metalinsulator transition point, the ground state will be an AFI. With a sufficient doping, Hubbard model becomes phase separated which is consistent with experimental results[4]. The obtained in the VCA phase separation in square lattice resembles an abrupt behavior of electron density near half-filling in the Hubbard model using the mean field and random phase approximations in one dimension and two-dimensional square lattice[41-43].

The phase diagram in Fig. 4 was calculated based on the assumption that short-range correlation is more important than long-range correlation, which is only valid when $U$ is small. At large $U$ the long-range antiferromagnetic correlations become essential and our assumption on the absence of the longrange order might not be applicable. It is hard to predict at what $U$ value the 


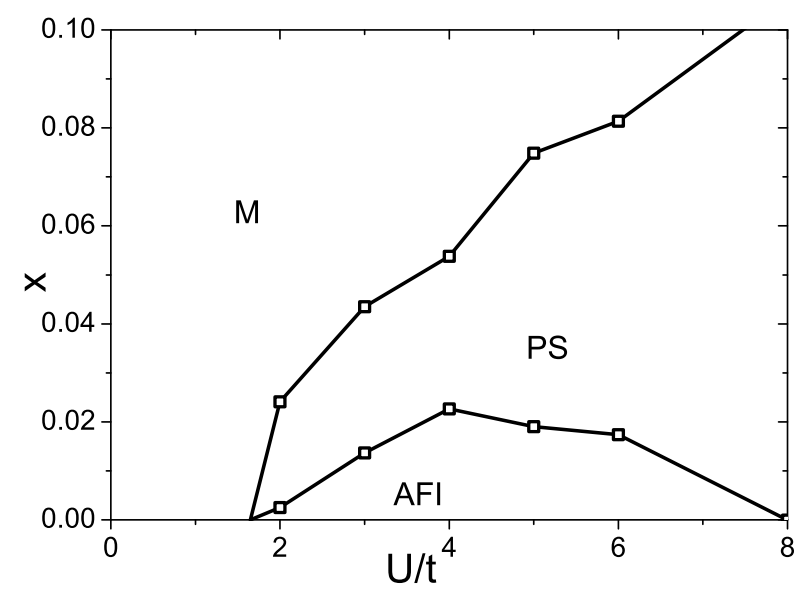

Fig. 4. Phase diagram of the Hubbard model in the square lattice. The phase diagram is drawn in the space of Coulomb interaction $U$ vs hole concentration $x=1-n$. In the figure, $\mathrm{M}$ stands for a metallic phase, which exists when $U$ is relatively low or the doping level is high. The AFI denotes an antiferromagnetic insulator, which exists at moderate $U$ values and low doping. PS denotes the phase separation region where AFI and M states are mixed and distributed inhomogeneously in the lattice. The results are calculated from VCA method using a $2 \times 2$ cluster as the reference system.

long-range ordering become important. Since the interaction beyond $U>8$ is generally considered strong enough to produce a significant long-range AF ordering $[20]$ and the results from the VCA without the introduction of a Weiss field may not be that reliable for such cases.

\subsubsection{Spectral function}

In order to further study the electronic properties of Hubbard model at phase separation, we use the VCA-based one-particle (excitation) spectral function to help understand the physics of phase separation. The one-particle (excitation) spectral function $A(\boldsymbol{k}, \omega)$ is obtained from the (imaginary part of the) one-particle Green's function $G(\boldsymbol{k}, \omega)$ as

$$
A(\boldsymbol{k}, \omega)=-2 \lim _{\eta \rightarrow 0^{+}} \Im G(\boldsymbol{k}, \omega+i \eta)
$$

where $\boldsymbol{k}$ is the $k$-point in the momentum space and $\omega$ is the real frequency. $\eta$ is a small parameter referred to as the spectrum broadening factor and it is set to 0.015 throughout the calculation. We extracted the spectral function for the two densities right before and after the electronic phase separation. The single particle excitation spectra at $n=0.977$ and $n=0.946$ with $U=4$ are shown in Fig. 5. By comparing the spectral functions at the above two densities, one can find that a set of narrow spectra (enclosed by a blue rectangle in Fig. 5) around the Fermi level disappears when the electron density drops from 0.977 
to 0.946 , i.e., this part of spectra at the Fermi level shows up in the AF state but disappears in the metallic state. This means that there is an instability of Fermi surface as phase separation occurs.

The part of spectra shown in Fig. 5 is narrow. The narrow band suggests the presence of relatively strong effective Coulomb interactions and hence electrons need extra energy to stay with other electrons in the state. The energy of the system is expected to increase when it approaches half-filling because more electrons are introduced to the narrow state. However, the local AF correlations also appear near half-filling which create a stable local magnetic structure which tends to decrease the total free energy of the system. These two competing effects (strong interactions and local AF correlations) give rise to an interesting situation: when the state is a mixture of a nearly half-filled state and an almost empty state, the total free energy is lower than a partially filled state with the same electron concentration. Therefore, the system energetically prefers redistributing over the lattice to form spatial charge variations, so that in some regions, the local electronic state is almost half-filled, while in other regions, the state is almost empty. This physical picture manifests the presence of a microscopic inhomogeneity, which achieves a lower total free energy than that of a uniform electron distribution. This result is similar to what our group[13-17] has predicted using calculations for isolated Hubbard clusters. For example, an ensemble of bipartite $2 \times 2$ square and $2 \times 4$ ladder clusters has a tendency to phase separation at moderate on-site Coulomb interaction when the energy of a mixture of electronic states with $N$ and $N-2$ electron configurations becomes lower than that of a pure two states with $N-1$ electrons. The vanishing of the above mentioned feature in the spectral function and with its occupied weight may be related to what was seen in the clusters; there is more gain in energy favoring the mixed (inhomogeneous) state at moderate $U$ as more holes have been added.

Another important point is that the position of this part of Fermi surface in the momentum space is the $(\pi / 2, \pi / 2)$ point (see the red arrow in Fig. 5 between the $\Gamma$ point and $M$ point and the red dots in Fig. $5(\mathrm{c})$ ). Because the unit cell of the AF state contains two sites, the first Brillouin zone (FBZ) of the AF state is folded and the boundary of FBZ is from $(0, \pi / 2)$ to $(\pi / 2,0)$. In the AF state, this part of Fermi surface resides on the FBZ boundary of the AF state. When the lattice becomes a metal, the unit cell only contains one site and the FBZ is unfolded, which causes a sudden change on FBZ boundaries (FBZ boundary deformation). The $(\pi / 2, \pi / 2)$ point is not on the FBZ boundary any more in the metallic state as shown in in Fig. 5(c). This part of Fermi surface at $(\pi / 2, \pi / 2)$ point also disappears. It seems the above Fermi surface instability is caused by the deformation of the FBZ boundary which is also related to the phase transition from an AF state to a metallic state. Similar conclusions were also predicted in Refs.[44,45] using the renormalization group (RG) method. The obtained peak in spectral density in the vicinity of the so called "hot 
spots" on the Fermi surface in momentum space in some extent resembles non-Fermi liquid like behavior, appearing at intersections of the Fermi surface with antiferromagnetic Brillouin zone boundary, which in the low temperature can lead to a significant Fermi surface reconstruction due to the doubling of the unit cell, similar to that appearing in the case of AFM long-range order in the pseudogap state [46]. Using the spectral functions of the Hubbard model, we can easily extract the density of states (DOS) of the system. However, since it involves an integral over k-points, certain fine features at k-points get somewhat clouded. Specific changes at k-points are not quite clear as in the case of the spectral functions and hence, we have not included the DOS plots in this paper.

It is interesting to investigate whether the spectral function feature discussed above is stable upon introduction of long-range orders. Comparing to studies by other groups, for example Ref [20], which includes long-range AF and SC orders to the VCA calculation of square lattice, Fig 5 in this reference also shows similar feature, which confirms the stability of this spectral feature.

\subsection{Honeycomb lattice}

Here it is interesting to contrast to results for square lattice and focus on phase separation in a honeycomb lattice $[28,29]$ since it has two sites per unit cell no matter whether it is in an AF or metallic state. In this circumstance a phase transition from an AF state to a metallic state is smooth, without deformation of FBZ boundary (See Fig. 6(c)). The one-particle spectral function for honeycomb lattice in Fig. 6 extracted from VCA calculations confirms that there is no instability at the Fermi surface.

The $n$ vs. $\mu$ plots for the honeycomb lattice are smooth for all the $U$ values and there is no sign of an electronic phase separation instability. This result demonstrates the geometry-driven character of deformation at the FBZ boundary.

\section{Summary}

We have studied possible electronic phase separation in the 2D Hubbard model for the bipartite square and honeycomb lattices using the VCA method. These calculations for the square lattice provide strong support for the existence of spontaneous phase separation which is a many-body effect closely related to 


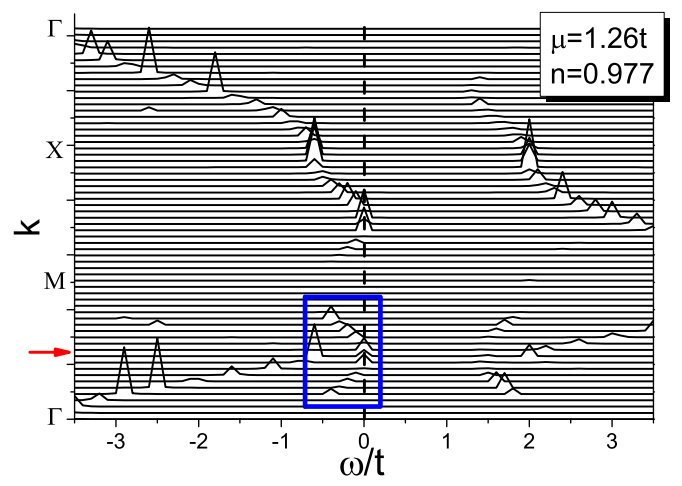

(a)

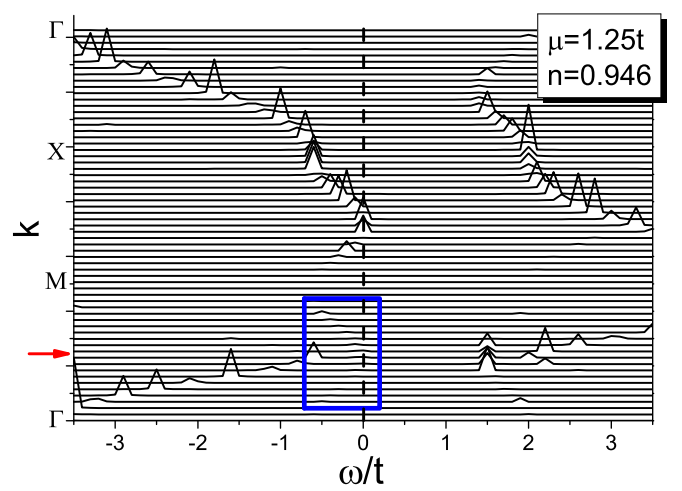

(b)

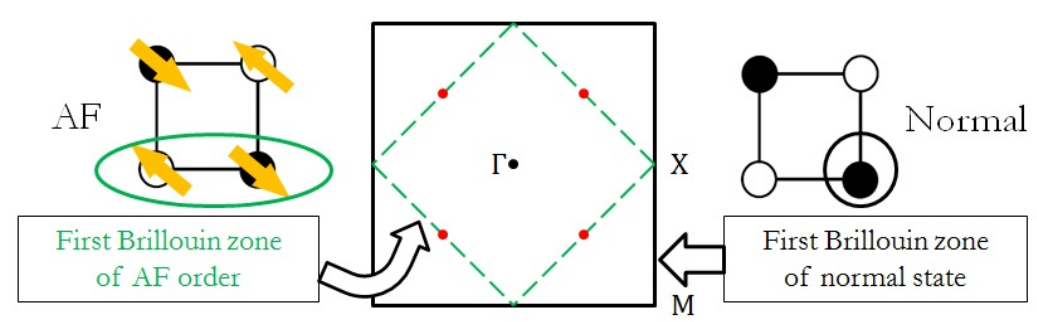

(c)

Fig. 5. One-particle excitation spectral functions for the Hubbard model on the square lattice right before and after phase separation are shown at (a) $\mu=1.26$ $(n=0.977)$ (b) $\mu=1.25(n=0.947)$. The dotted line at $\omega=0$ denotes the Fermi level. At the higher electron density $(n=0.977)$, a part of the spectra in (a), which is enclosed in the blue square, shows up around $(\pi / 2, \pi / 2)$ point (marked by red arrow) at the Fermi level, but it vanishes in (b) at the lower density $n=0.947$ (almost no peaks in the blue square). (c) shows the first Brillouin zone (FBZ) of a square lattice and the $k$-points shown in (a) and (b) are also marked. The FBZs for the AF state and the normal state are different as shown in this figure. The spectral functions are calculated from the VCA method using a 2x2 cluster as the reference system. 


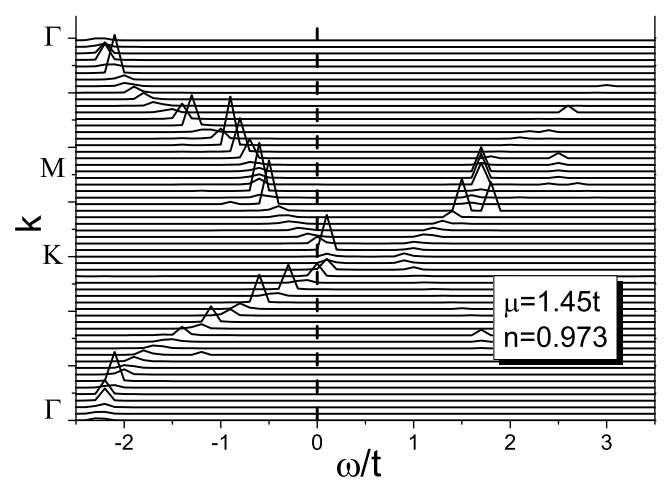

(a)

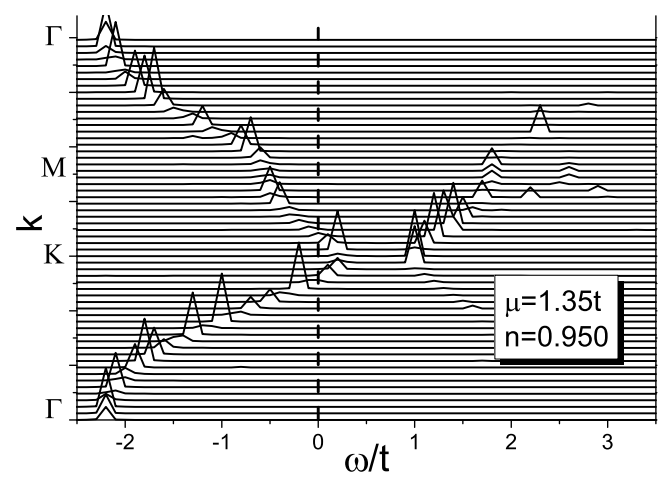

(b)

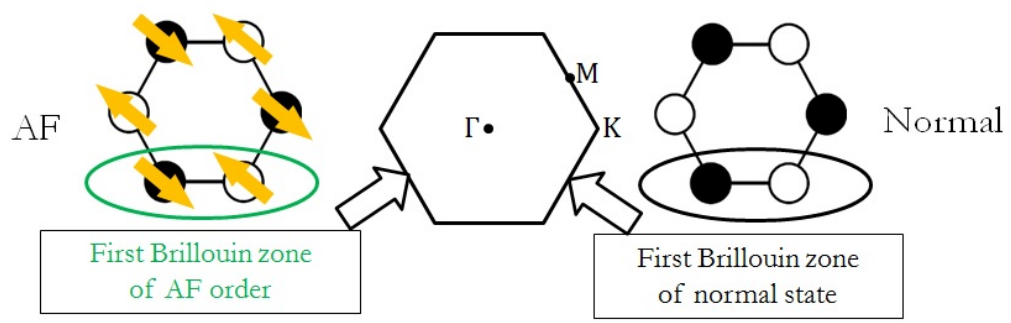

(c)

Fig. 6. One-particle excitation spectral functions for the Hubbard model on the honeycomb lattice with similar electron density with Fig. 5: (a) $\mu=1.45(n=0.970)$ (b) $\mu=1.35(n=0.950)$. The dotted line at $\omega=0$ denotes the Fermi level. The spectra do not change too much between two electron densities. The missing spectrum observed in the square lattice (Fig. 5) is not found in the honeycomb lattice, which implies that there is no instability of the Fermi surface. (c) shows the first Brillouin zone (FBZ) of a honeycomb lattice and the $k$-points shown in (a) and (b) are also marked. The FBZs for the AF state and the normal state are the same for the honeycomb lattice. The reference system of honeycomb lattice is shown in Fig. 7 . 


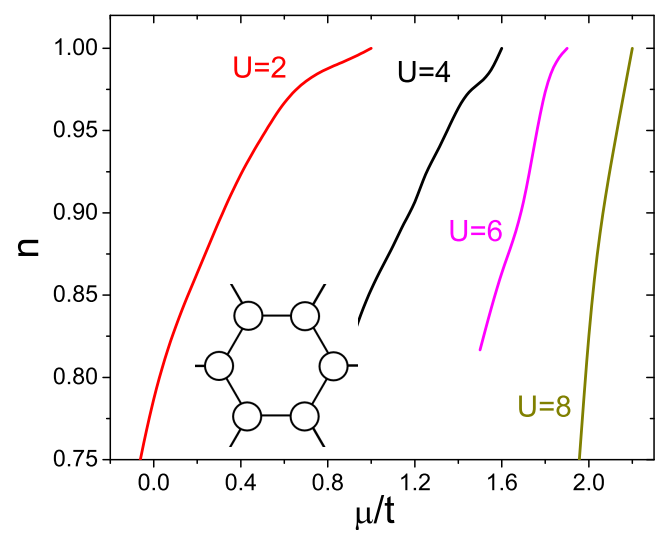

Fig. 7. The electron densities $n$ as a function of chemical potential $\mu$ for the Hubbard model on a honeycomb lattice at different values of on-site Coulomb interaction $U$. The plots for the honeycomb lattice are smooth without any discontinuities which implies that phase separation is absent in the honeycomb lattice. The reference system used in the VCA calculation is also shown here.

the AF transition at half-filling. Our previously published work, at the isolated quantum cluster level in Refs. [15,16], identified the above as being due to many-body level crossings at zero and finite temperature, favoring a mixed state when enough holes are added at moderate $U$. Thus, disconnected cluster study can capture some of the essential physics of phase separation and nanoscale inhomogeneities in large "concentrated" systems due to the local electron correlations and appropriate cluster geometry. The VCA method, which goes beyond the aforementioned cluster work and introduces clustercluster interactions using a variational approach, provide substantial support for the phase separation tendency obtained earlier; i.e., spontaneous phase separation observed in small square clusters persists also in the larger size systems. In particular, it is possible to identify differences in the spectral function and spin susceptibilities away from half-filling. The electronic phase separation, at least in part, might be a result of a Fermi surface instability which can be tied to a geometry-related deformation of the FBZ boundary: during the transition of the square lattice from an AF to a metallic state, the number of lattice sites per unit cell will change from 2 to 1 causing a deformation of the FBZ boundary. The vanishing of this feature with its occupied weight of the spectral function can be related to what was seen in the clusters; there is a gain in energy favoring the mixed (inhomogeneous) state as enough holes are added. For the honeycomb lattice, there is no such deformation of the FBZ boundary and our study of the honeycomb lattice confirms the absence of phase separation near half-filling. 


\section{Acknowledgements}

The authors acknowledge Jian-Xin Zhu and the computing facilities provided by the Center for Integrated Nanotechnologies, a U.S. Department of Energy, Office of Basic Energy Sciences user facility at Los Alamos National Laboratory (Contract DE-AC52-06NA25396) and Sandia National Laboratories (Contract DE-AC04-94AL85000). The work was performed also, in part, at the Center for Functional Nanomaterials, Brookhaven National Laboratory supported by the U.S. Department of Energy, Office of Basic Energy Sciences, under Contract No.DE-AC02-98CH10886.

\section{References}

[1] J. M. Tranquada, B. J. Sternlieb, J. D. Axe, Y. Nakamura, S. Uchida, Evidence for stripe correlations of spins and holes in copper oxide superconductors, Nature 375 (1995) 561-563. doi:10.1038/375561a0.

[2] J. E. Hoffman, E. W. Hudson, K. M. Lang, V. Madhavan, H. Eisaki, S. Uchida, J. C. Davis, A four unit cell periodic pattern of quasi-particle states surrounding vortex cores in bi2sr2cacu2o8+, Science 295 (5554) (2002) 466-469. doi:10.1126/science.1066974.

[3] K. M. Lang, V. Madhavan, J. E. Hoffman, E. W. Hudson, H. Eisaki, S. Uchida, J. C. Davis, Imaging the granular structure of high- $\mathrm{T}_{c}$ superconductivity in underdoped $\mathrm{Bi}_{2} \mathrm{Sr}_{2} \mathrm{CaCu}_{2} \mathrm{O}_{8+}$, Nature 415 (2002) 412-416. doi:10.1038/415412a.

[4] H. E. Mohottala, B. O. Wells, J. I. Budnick, W. A. Hines, C. Niedermayer, L. Udby, C. Bernhard, A. R. Moodenbaugh, F.-C. Chou, Phase separation in superoxygenated $\mathrm{La}_{2-x} \mathrm{Sr}_{x} \mathrm{CuO}_{4+y}$, Nature Materials 5 (2006) 377-382. doi:10.1038/nmat1633.

[5] J. Hubbard, Electron correlations in narrow energy bands, Royal Society of London Proceedings Series A 276 (1963) 238-257. doi:10.1098/rspa.1963.0204.

[6] M. Jarrell, Hubbard model in infinite dimensions: A quantum monte carlo study, Phys. Rev. Lett. 69 (1992) 168-171. doi:10.1103/PhysRevLett.69.168.

[7] A. Georges, G. Kotliar, W. Krauth, M. J. Rozenberg, Dynamical mean-field theory of strongly correlated fermion systems and the limit of infinite dimensions, Rev. Mod. Phys. 68 (1996) 13125. doi:10.1103/RevModPhys.68.13.

[8] T. Maier, M. Jarrell, T. Pruschke, M. H. Hettler, Quantum cluster theories, Rev. Mod. Phys. 77 (2005) 1027-1080. doi:10.1103/RevModPhys.77.1027. 
[9] M. Potthoff, M. Aichhorn, C. Dahnken, Variational cluster approach to correlated electron systems in low dimensions, Phys. Rev. Lett. 91 (2003) 206402. doi:10.1103/PhysRevLett.91.206402.

[10] P. B. Visscher, Phase separation instability in the hubbard model, Phys. Rev. B 10 (1974) 943-945. doi:10.1103/PhysRevB.10.943.

[11] V. J. Emery, S. A. Kivelson, H. Q. Lin, Phase separation in the $t-J$ model, Phys. Rev. Lett. 64 (1990) 475-478. doi:10.1103/PhysRevLett.64.475.

[12] V. Emery, S. Kivelson, Frustrated electronic phase separation and hightemperature superconductors, Physica C: Superconductivity 209 (4) (1993) 597-621. doi:10.1016/0921-4534(93)90581-A.

[13] A. N. Kocharian, G. W. Fernando, K. Palandage, J. W. Davenport, Exact study of charge-spin separation, pairing fluctuations, and pseudogaps in four-site hubbard clusters, Phys. Rev. B 74 (2006) 024511. doi:10.1103/PhysRevB.74.024511.

[14] G. W. Fernando, A. N. Kocharian, K. Palandage, T. Wang, J. W. Davenport, Phase separation and electron pairing in repulsive hubbard clusters, Phys. Rev. B 75 (2007) 085109. doi:10.1103/PhysRevB.75.085109.

[15] A. N. Kocharian, G. W. Fernando, K. Palandage, J. W. Davenport, Coherent and incoherent pairing instabilities and spin-charge separation in bipartite and nonbipartite nanoclusters: Exact results, Phys. Rev. B 78 (2008) 075431. doi:10.1103/PhysRevB.78.075431.

[16] G. W. Fernando, K. Palandage, A. N. Kocharian, J. W. Davenport, Pairing in bipartite and nonbipartite repulsive hubbard clusters: Octahedron, Phys. Rev. B 80 (2009) 014525. doi:10.1103/PhysRevB.80.014525.

[17] K. Fang, G. W. Fernando, A. N. Kocharian, Pairing enhancement in betts lattices with next nearest neighbor couplings: Exact results, Physics Letters A 376 (4) (2012) 538 - 543. doi:10.1016/j.physleta.2011.11.026.

[18] A. Macridin, M. Jarrell, T. Maier, Phase separation in the hubbard model using the dynamical cluster approximation, Phys. Rev. B 74 (2006) 085104. doi:10.1103/PhysRevB.74.085104.

[19] E. Khatami, K. Mikelsons, D. Galanakis, A. Macridin, J. Moreno, R. T. Scalettar, M. Jarrell, Quantum criticality due to incipient phase separation in the two-dimensional hubbard model, Phys. Rev. B 81 (2010) 201101. doi:10.1103/PhysRevB.81.201101.

[20] M. Aichhorn, E. Arrigoni, M. Potthoff, W. Hanke, Variational cluster approach to the hubbard model: Phase-separation tendency and finite-size effects, Phys. Rev. B 74 (2006) 235117. doi:10.1103/PhysRevB.74.235117.

[21] M. Aichhorn, E. Arrigoni, M. Potthoff, W. Hanke, Phase separation and competition of superconductivity and magnetism in the two-dimensional hubbard model: From strong to weak coupling, Phys. Rev. B 76 (2007) 224509. doi:10.1103/PhysRevB.76.224509. 
[22] R. S. Markiewicz, Phase separation near the mott transition in la 2-x sr x cuo 4, Journal of Physics: Condensed Matter 2 (3) (1990) 665.

[23] M. Capone, G. Sangiovanni, C. Castellani, C. Di Castro, M. Grilli, Phase separation close to the density-driven mott transition in the hubbard-holstein model, Phys. Rev. Lett. 92 (2004) 106401. doi:10.1103/PhysRevLett.92.106401.

[24] B. V. Fine, T. Egami, Phase separation in the vicinity of quantum-critical doping concentration: Implications for high-temperature superconductors, Phys. Rev. B 77 (2008) 014519. doi:10.1103/PhysRevB.77.014519.

[25] Y. Z. Zhang, M. Imada, Pseudogap and mott transition studied by cellular dynamical mean-field theory, Phys. Rev. B 76 (2007) 045108. doi:10.1103/PhysRevB.76.045108.

[26] M. Balzer, B. Kyung, D. Snchal, A.-M. S. Tremblay, M. Potthoff, First-order mott transition at zero temperature in two dimensions: Variational plaquette study, EPL (Europhysics Letters) 85 (1) (2009) 17002.

[27] M. Vekić, S. R. White, Pseudogap formation in the half-filled hubbard model, Phys. Rev. B 47 (1993) 1160-1163. doi:10.1103/PhysRevB.47.1160.

[28] S. Sorella, E. Tosatti, Semi-metal-insulator transition of the hubbard model in the honeycomb lattice, EPL (Europhysics Letters) 19 (8) (1992) 699.

[29] M.-T. Tran, K. Kuroki, Finite-temperature semimetal-insulator transition on the honeycomb lattice, Phys. Rev. B 79 (2009) 125125. doi:10.1103/PhysRevB.79.125125.

[30] G. Santoro, M. Airoldi, S. Sorella, E. Tosatti, Hubbard model on the infinite-dimensional diamond lattice, Phys. Rev. B 47 (1993) 16216-16221. doi:10.1103/PhysRevB.47.16216.

[31] A. Moreo, D. Scalapino, E. Daggato, Phase separation in the hubbard model, Phys. Rev. B 43 (1991) 11442-11444. doi:10.1103/PhysRevB.43.11442.

[32] F. Becca, M. Capone, S. Sorella, Spatially homogeneous ground state of the two-dimensional hubbard model, Phys. Rev. B 62 (2000) 12700-12706. doi:10.1103/PhysRevB.62.12700.

[33] G. Su, Phase separation in the two-dimensional hubbard model, Phys. Rev. B 54 (1996) R8281-R8284. doi:10.1103/PhysRevB.54.R8281.

[34] D. D. Betts, S. Masui, N. Vats, G. E. Stewart, Improved finite-lattice method for estimating the zero-temperature properties of two-dimensional lattice models, Canadian Journal of Physics 74 (1-2) (1996) 54-64. doi:10.1139/p96-010.

URL http://dx.doi.org/10.1139/p96-010

[35] D. D. Betts, H. Q. Lin, J. Flynn, Improved finite-lattice estimates of the properties of two quantum spin models on the infinite square lattice, Canadian Journal of Physics 77 (5) (1999) 353-369. doi:10.1139/Canad.J.Phys./p99-041. 
[36] M. Potthoff, Self-energy-functional approach to systems of correlated electrons, The European Physical Journal B - Condensed Matter and Complex Systems 32 (4) (2003) 429-436. doi:10.1140/epjb/e2003-00121-8.

[37] J. Cullum, R. Willoughby, Lanczos algorithms for large symmetric eigenvalue computations: Volume 1, Theory, Classics in Applied Mathematics, Society for Industrial and Applied Mathematics, 2002.

[38] D. Sénéchal, D. Perez, M. Pioro-Ladrière, Spectral weight of the hubbard model through cluster perturbation theory, Phys. Rev. Lett. 84 (2000) 522525. doi:10.1103/PhysRevLett.84.522.

[39] D. Sénéchal, D. Perez, D. Plouffe, Cluster perturbation theory for hubbard models, Phys. Rev. B 66 (2002) 075129. doi:10.1103/PhysRevB.66.075129.

[40] L. Landau, E. Lifshitz, Statistical Physics, no. v. 5, Elsevier Science, 1996.

[41] H. Yamase, V. Oganesyan, W. Metzner, Mean-field theory for symmetrybreaking fermi surface deformations on a square lattice, Phys. Rev. B 72 (2005) 035114. doi:10.1103/PhysRevB.72.035114.

URL http://link.aps.org/doi/10.1103/PhysRevB.72.035114

[42] A. N. Kocharian, C. Yang, Y. L. Chiang, T. Y. Chou, Exact and self-consistent results in one-dimensional repulsive hubbard model, International Journal of Modern Physics B 17 (30) (2003) 5749-5772. arXiv:http://www.worldscientific.com/doi/pdf/10.1142/S0217979203023380, doi:10.1142/S0217979203023380.

URL http://www. worldscientific.com/doi/abs/10.1142/S0217979203023380

[43] A. N. Andriotis, E. N. Economou, Q. Li, C. M. Soukoulis, Phase separation in the hubbard model, Phys. Rev. B 47 (1993) 9208-9214. doi:10.1103/PhysRevB.47.9208.

URL http://link.aps.org/doi/10.1103/PhysRevB.47.9208

[44] N. Furukawa, T. M. Rice, M. Salmhofer, Truncation of a two-dimensional fermi durface due to quasiparticle gap formation at the saddle points, Phys. Rev. Lett. 81 (1998) 3195-3198. doi:10.1103/PhysRevLett.81.3195.

[45] N. Furukawa, T. M. Rice, Instability of a landau - fermi liquid as the mott insulator is approached, Journal of Physics: Condensed Matter 10 (23) (1998) L381.

[46] E. Z. Kuchinskii, M. V. Sadovskii, Reconstruction of the fermi surface in the pseudogap state of cuprates, JETP Lett. 88 (2008) 192-196. doi:10.1134/S0021364008150101. 


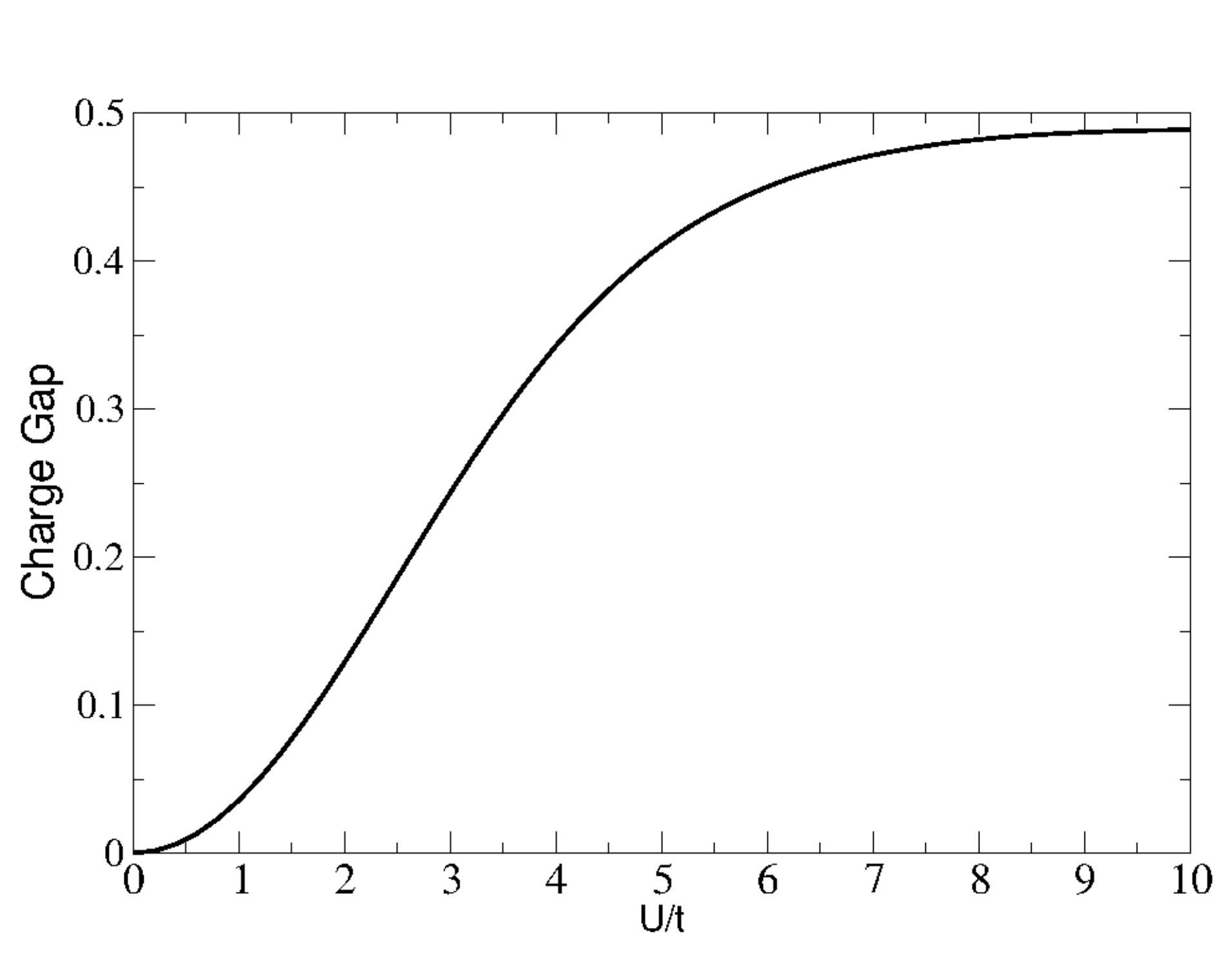

Figure

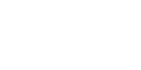

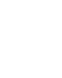

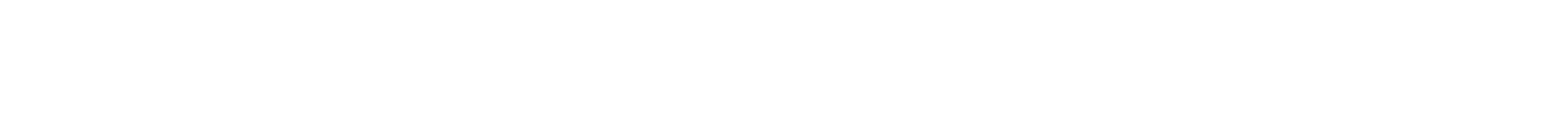




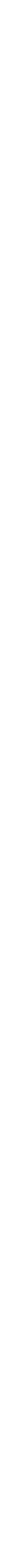

Figure

.

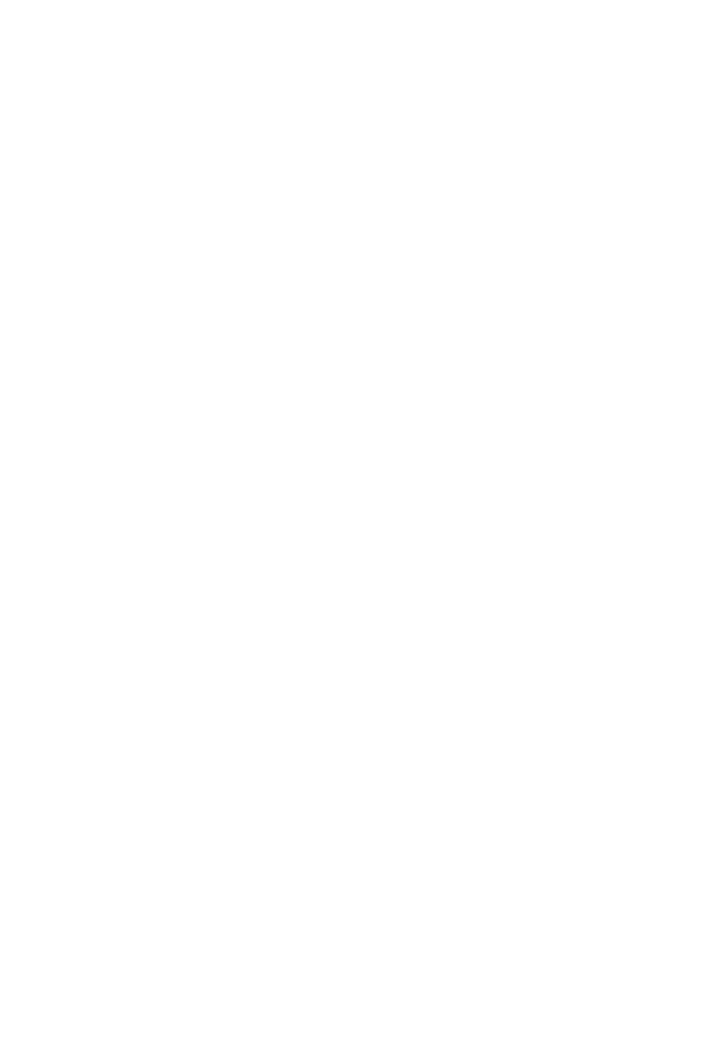




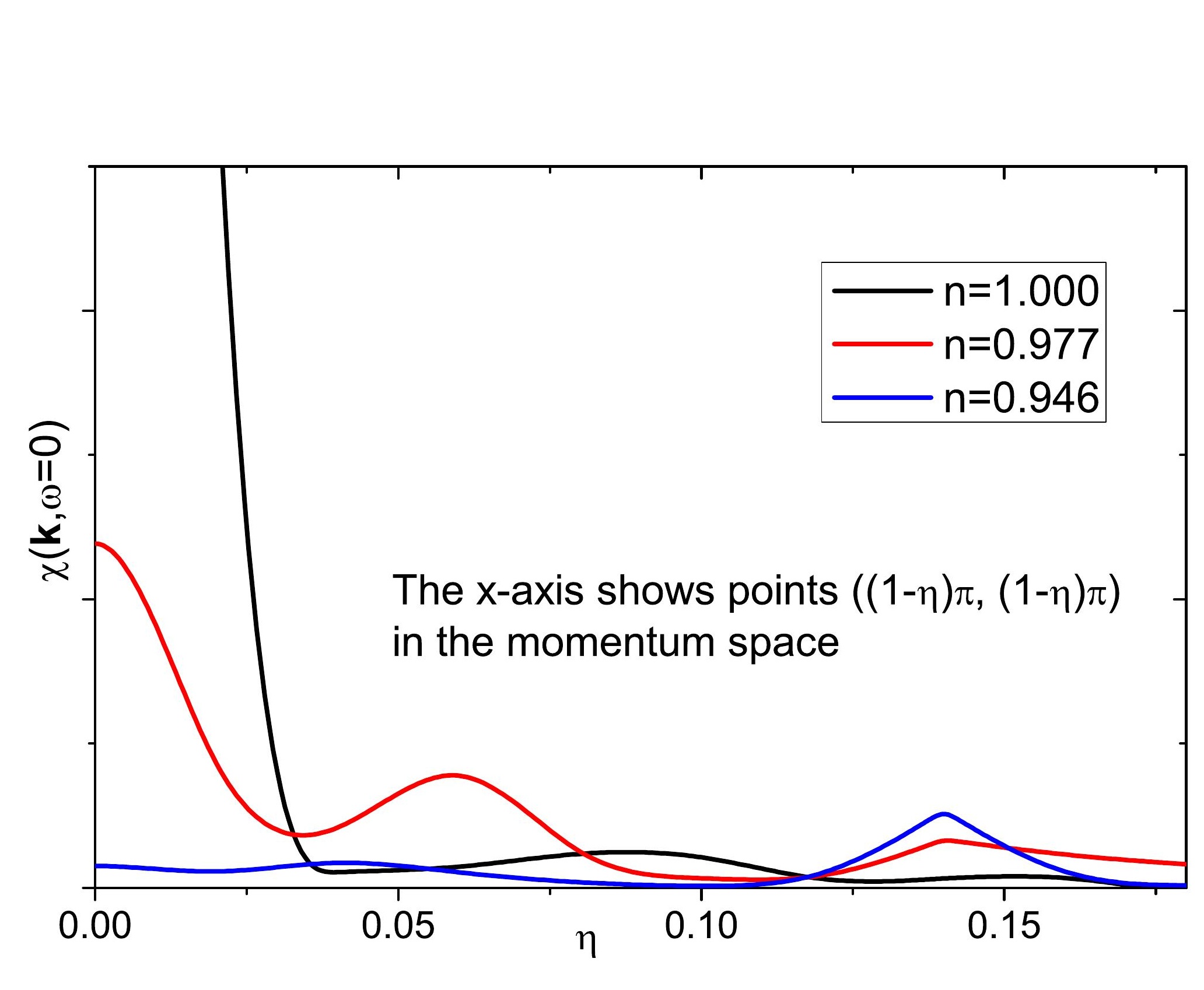

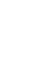
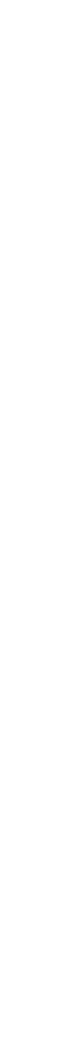


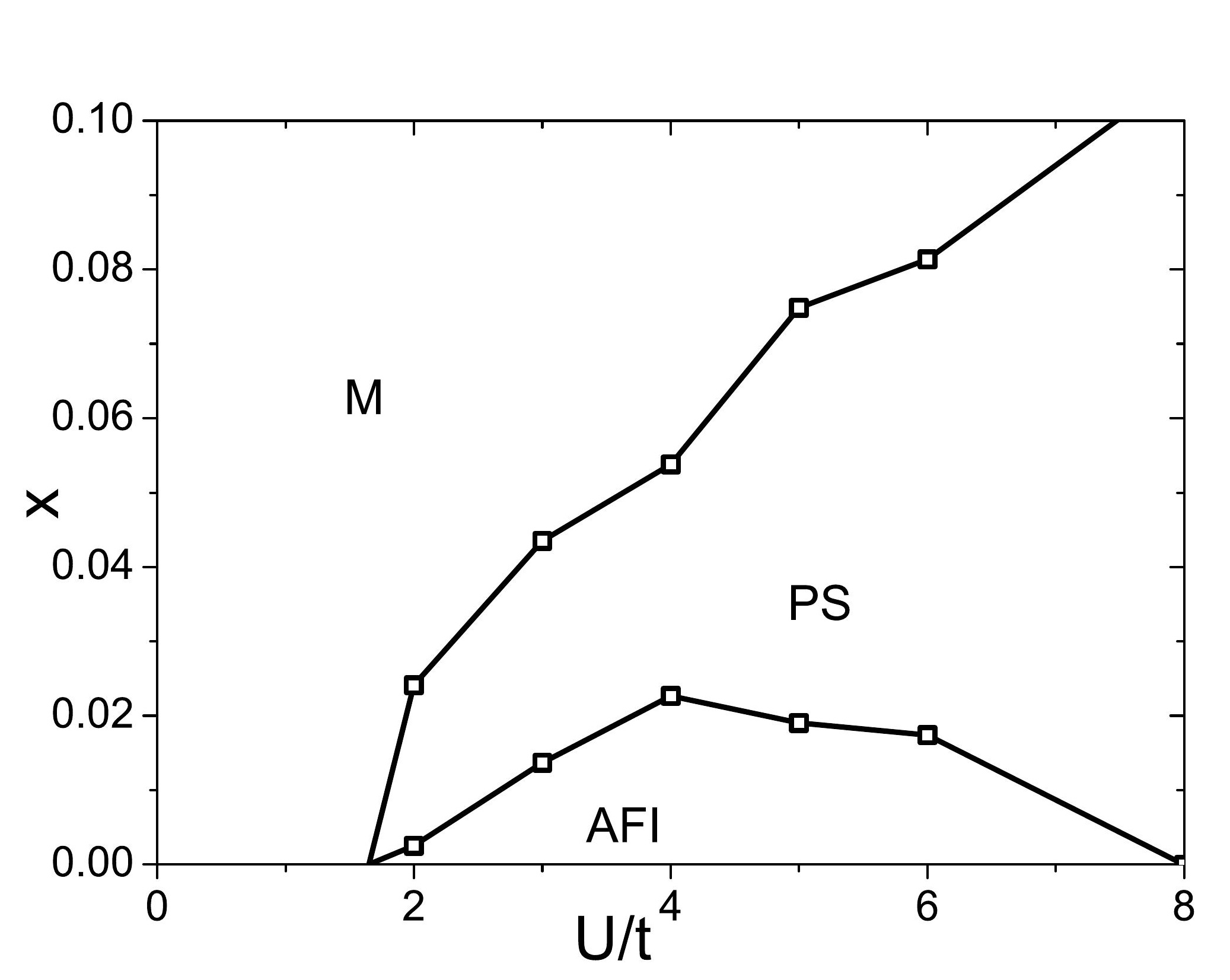

Figure

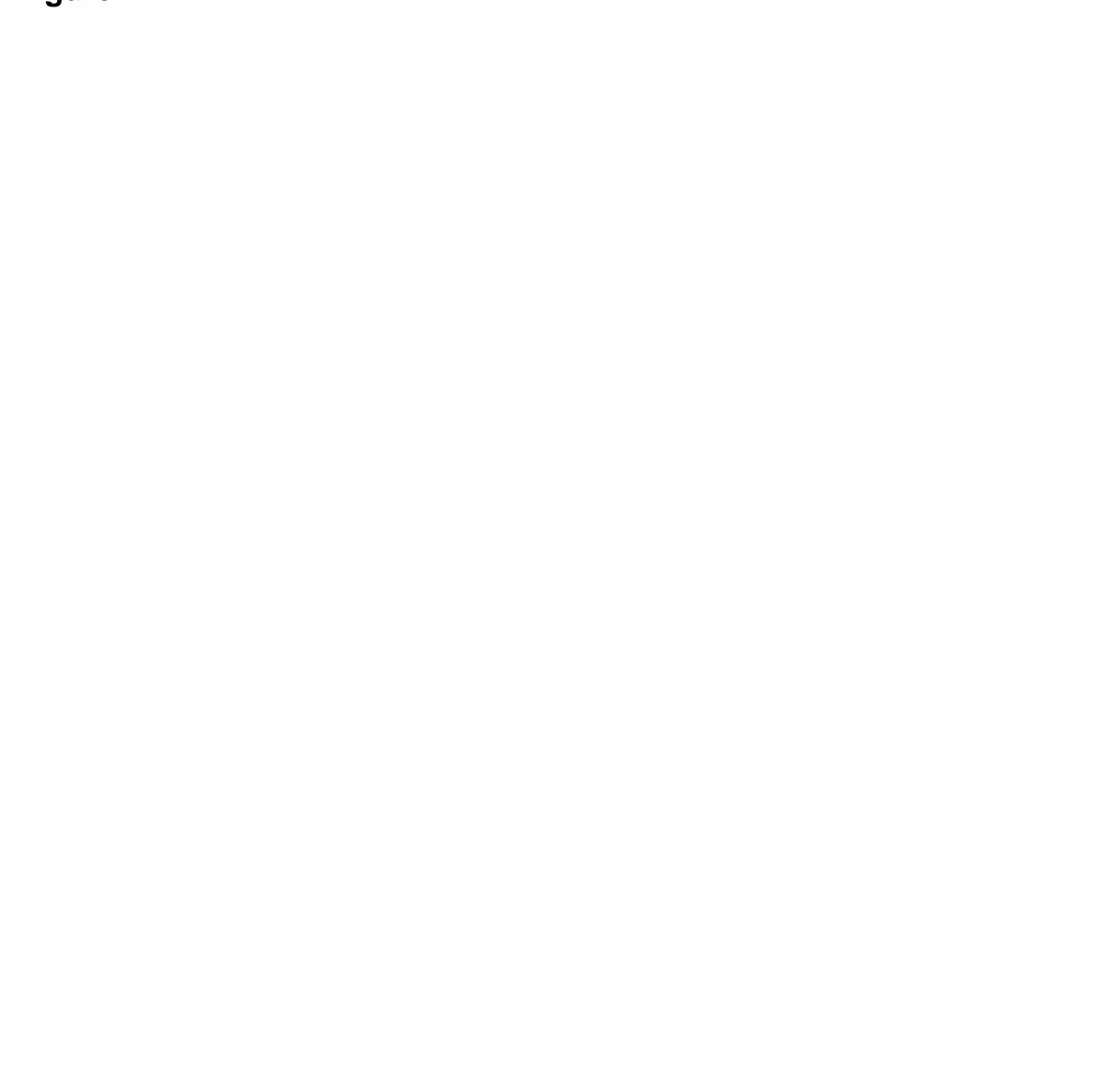




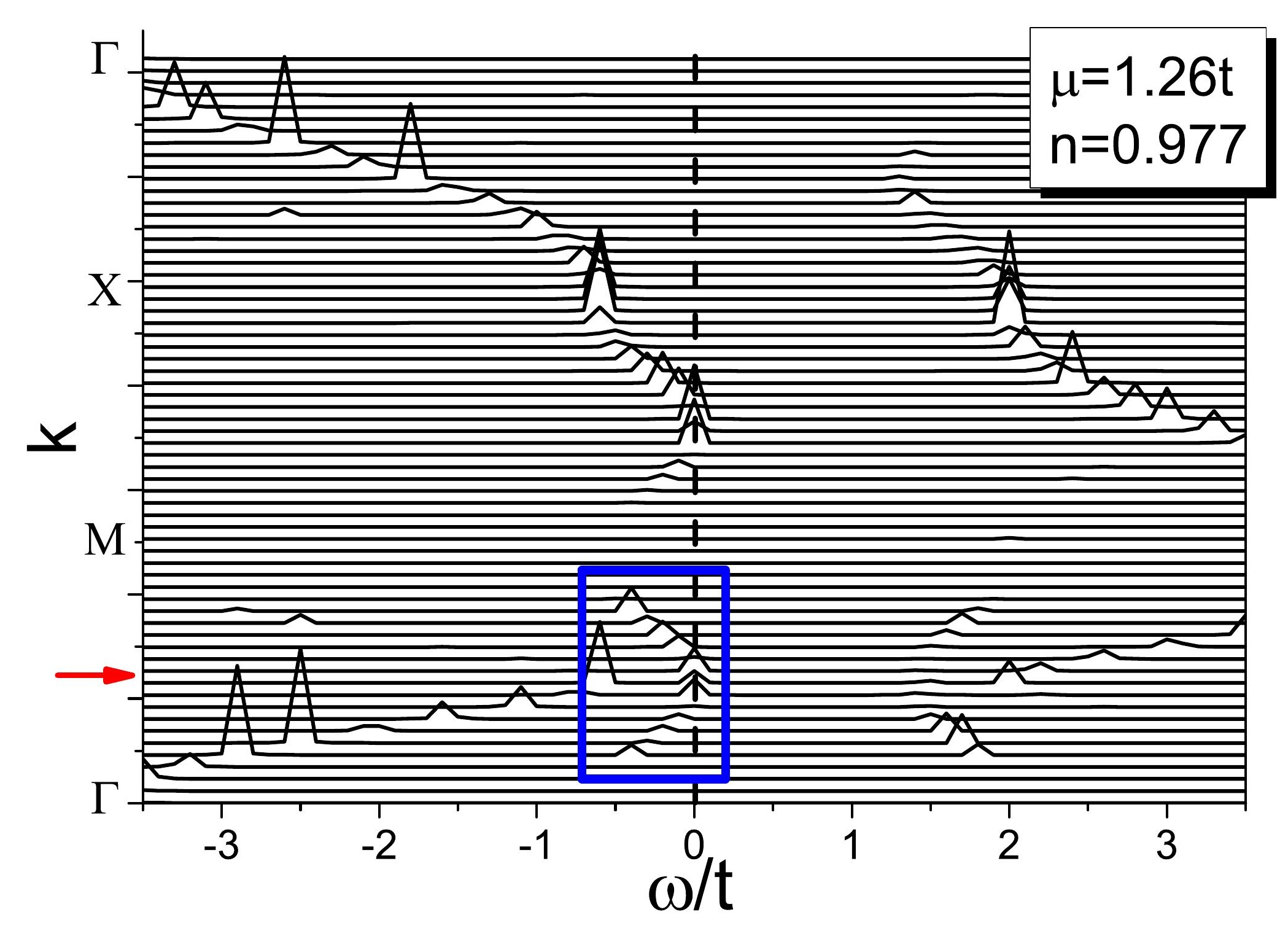

Figure
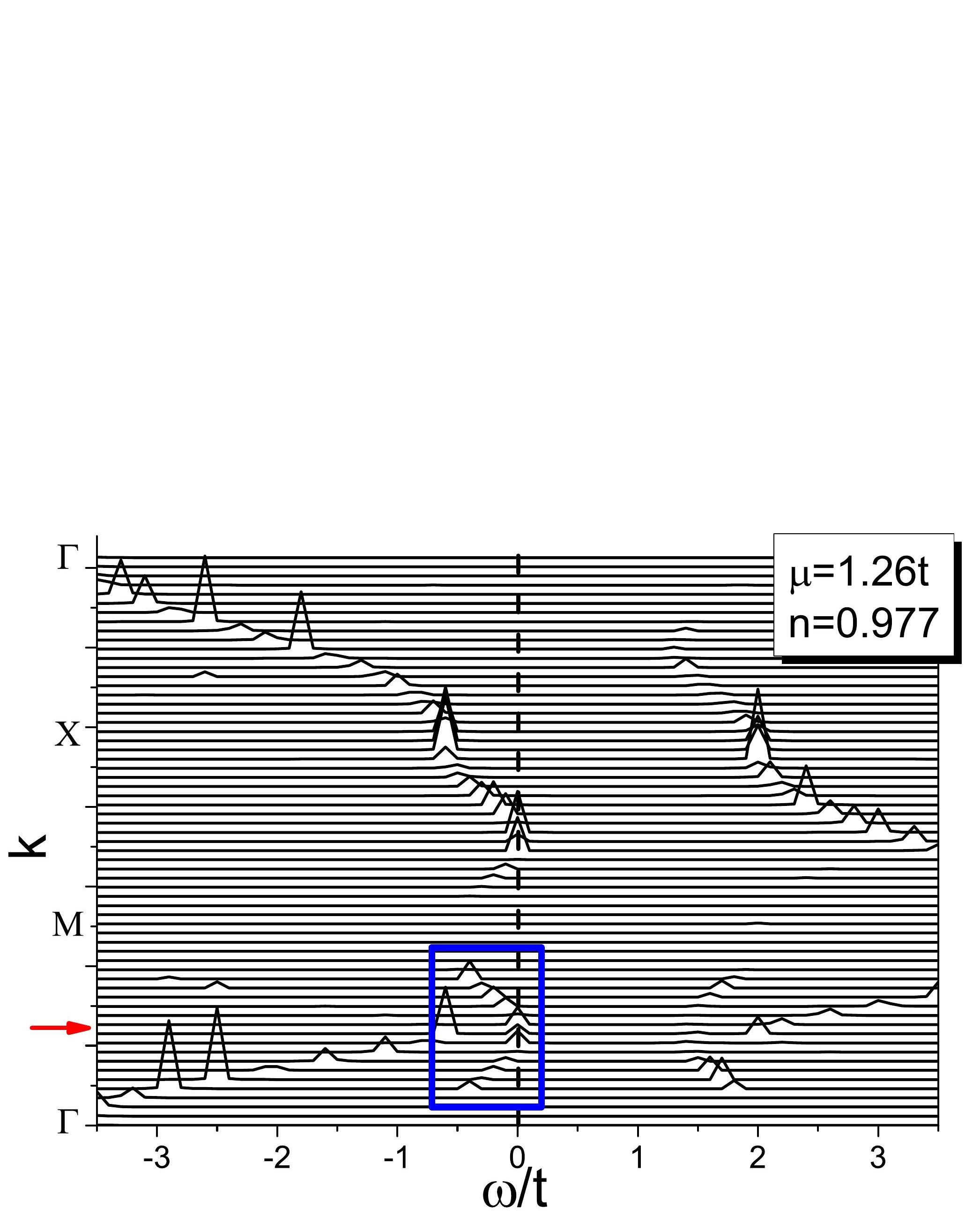


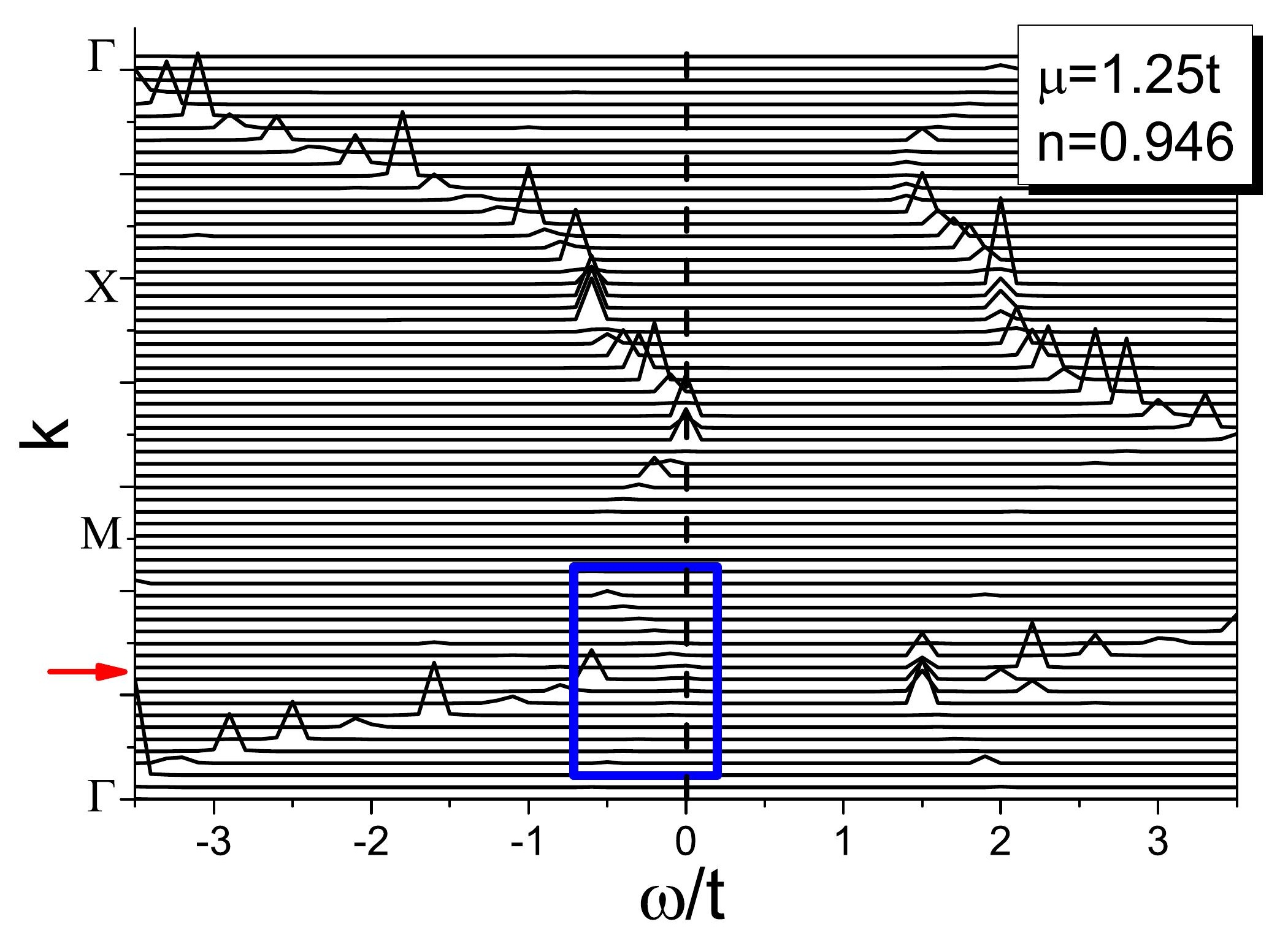




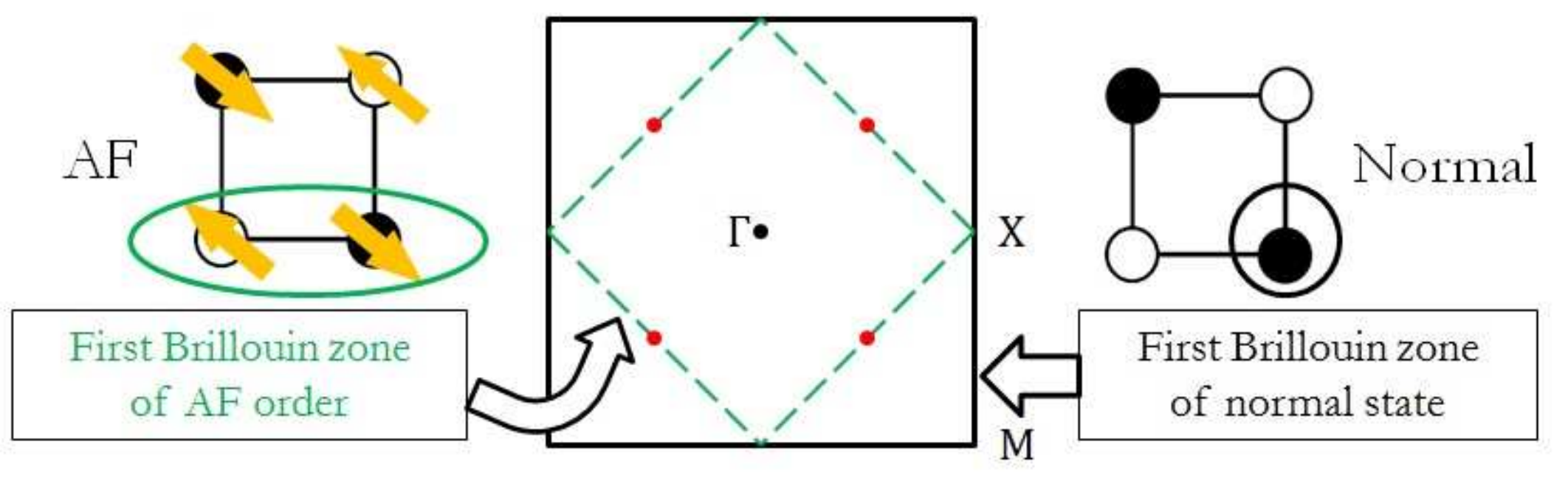

Figure 


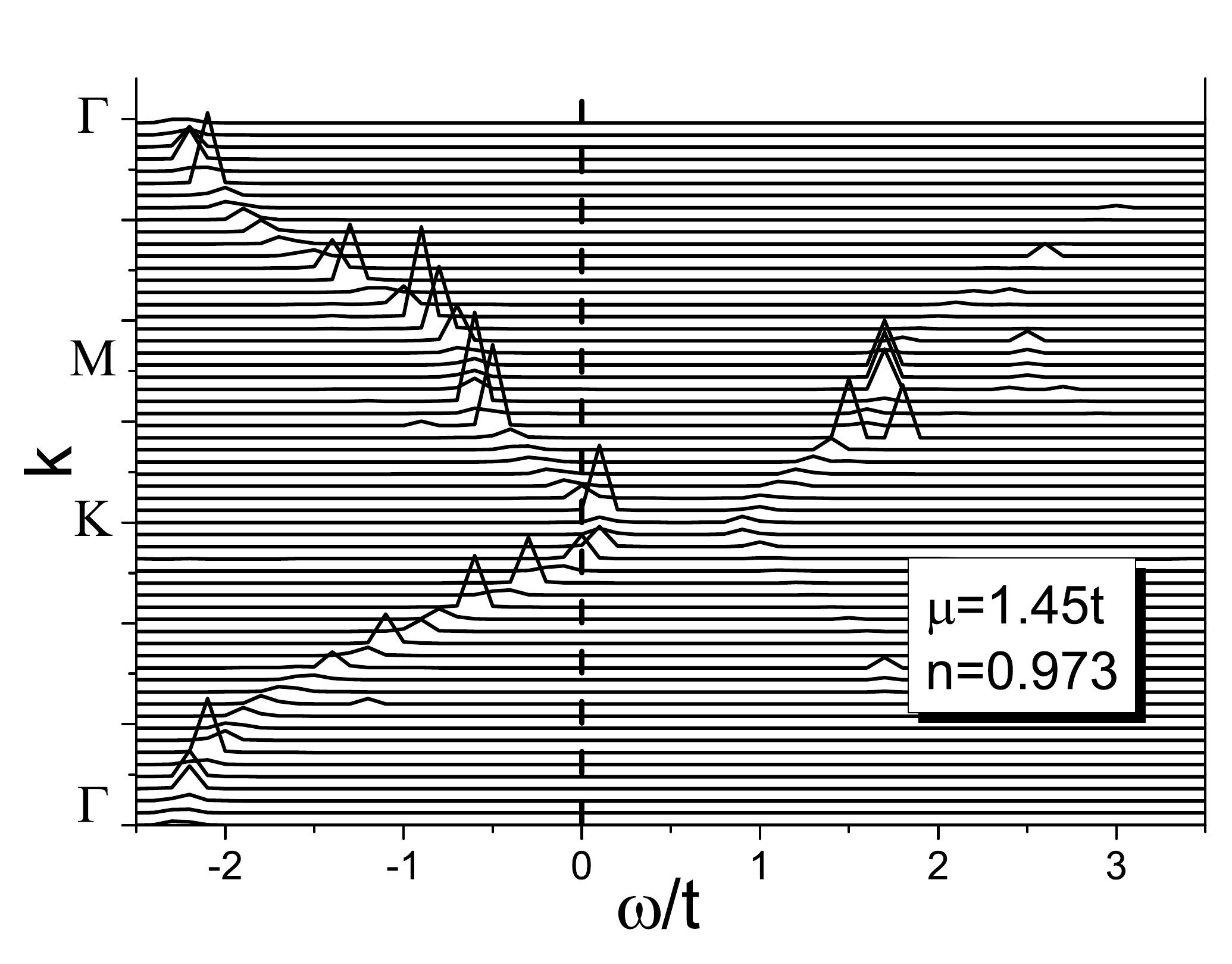

Figure 


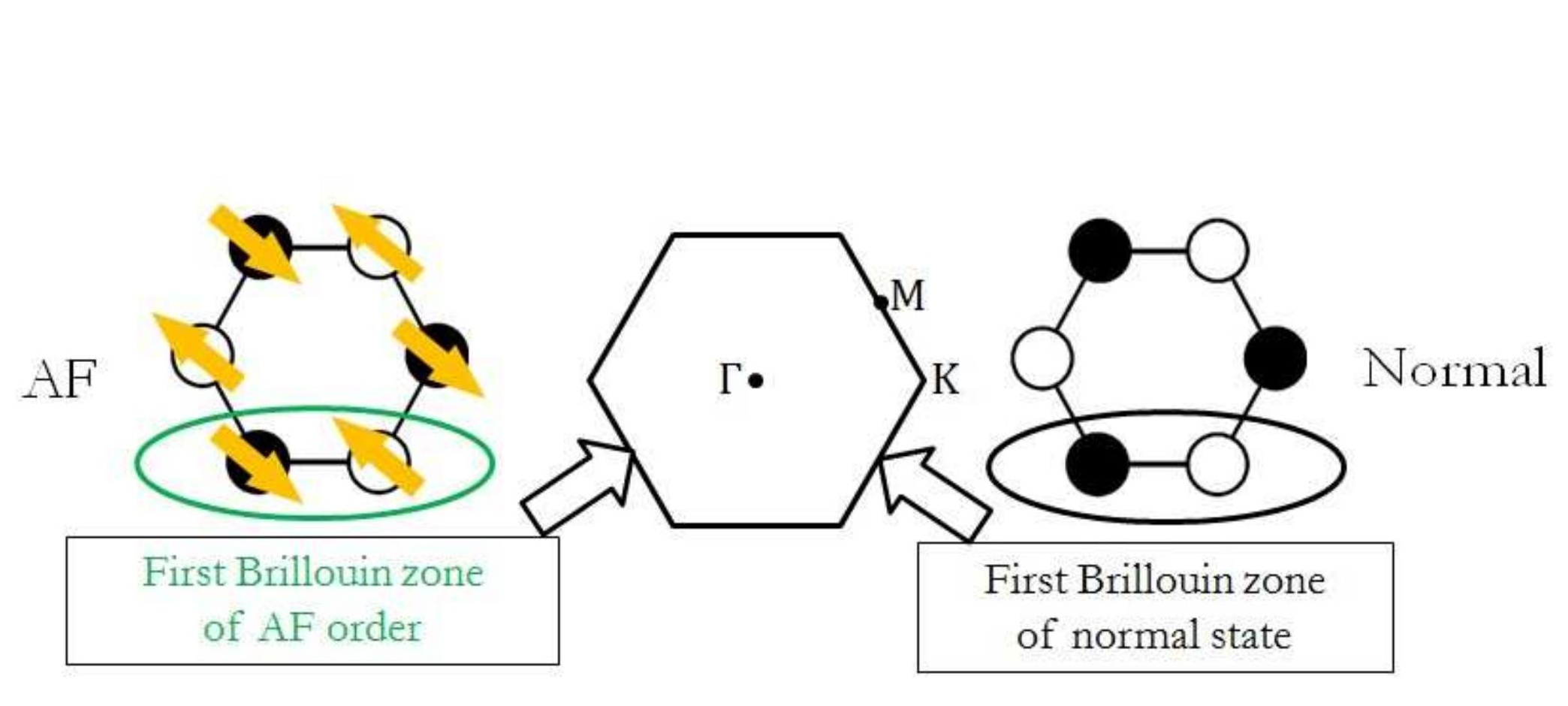

Figure

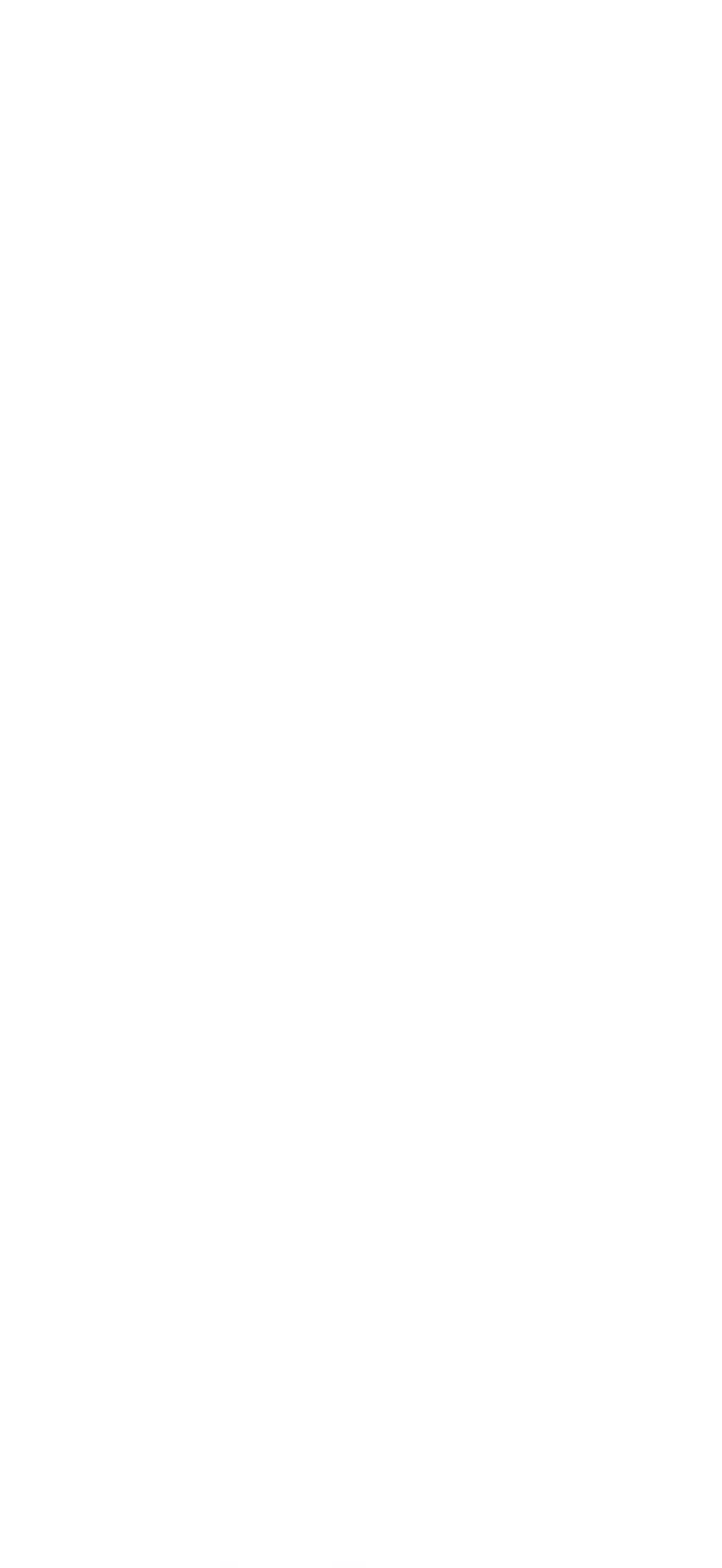

